\title{
Epigenetic effects of riboflavin supplementation on hypertension in adults screened for the MTHFR C677 T polymorphism
}

The C677 $\mathrm{T}$ polymorphism in the folate metabolising enzyme methylenetetrahydrofolate reductase (MTHFR) is associated with hypertension $^{(1)}$. Riboflavin acts as a cofactor for MTHFR in one-carbon metabolism which generates methyl groups that are utilised in important biological reactions such as DNA methylation ${ }^{(2)}$. Supplementation with riboflavin has previously been shown to lower blood pressure in individuals homozygous for the MTHFR 677TT genotype ${ }^{(3,4)}$. The mechanism linking this gene-nutrient interaction is currently unknown but may involve aberrant DNA methylation, which has been implicated in hypertension ${ }^{(5)}$. Therefore, the aims of this study were to examine global and $M T H F R$ gene DNA methylation in adults stratified by $M T H F R$ genotype and in response to intervention with $1.6 \mathrm{mg}$ /day of riboflavin specifically in individuals with the MTHFR 677TT genotype.

Stored peripheral blood leukocyte samples from participants screened for the MTHFR C677 T genotype who had consented and participated in targeted RCTs $(1.6 \mathrm{mg} /$ riboflavin/day for 16 weeks) at the Ulster University Nutrition Innovation Centre for Food and Health (NICHE) were accessed for this study $(n=120)$. Extracted DNA was bisulphite converted and pyrosequencing was used to analyse DNA methylation. One-way ANCOVA and within-between repeated measures (adjusting for age, gender and smoking status) were used to analyse baseline and DNA methylation differences in response to intervention respectively.

LINE-1 and MTHFR north shore DNA methylation were not different between the MTHFR C677 T genotype groups at baseline however subsequent supplementation with riboflavin resulted in a decrease of $(-3.2 \% ; p<0 \cdot 018)$ in LINE-1 and $(-1 \cdot 2 \% ; p<0 \cdot 001)$ in MTHFR north shore methylation in TT genotype participants. Participants with the TT genotype had significantly higher south shelf methylation $(+4.7 \%$; $<0.0001)$ compared to $\mathrm{CC}$ individuals at baseline but this was not influenced by subsequent supplementation with riboflavin. Changes in riboflavin biomarker status in response to intervention were associated with significant changes in LINE-1 ( $\mathrm{r}=0.270, \mathrm{p}<0.018)$ and $M T H F R$ north shore $(\mathrm{r}=0.414, \mathrm{p}<0.0001)$ methylation (TT genotype only).

This is the largest study so far to provide RCT evidence that DNA methylation can be modulated by riboflavin in adults with the MTHFR 677TT genotype. Riboflavin supplementation influenced global and MTHFR methylation in these genetically at-risk adults. Further work will provide insights into the mechanism of riboflavin action in lowering blood pressure in these genetically at-risk adults.

1. Yang B, Fan S, et al. (2014) Plos One 9, 1-13.

2. Yamada K, Chen Z, Rozen R, Matthews RG, et al. (2001) PNAS 98, 14853-8.

3. Wilson CP, McNulty H, Ward M, et al. (2013) Hypertension 61, 1302-1308.

4. Horigan G, McNulty H, Ward M, et al. (2010) J Hypertension 28, 478-486.

5. Muka T, Koromani F, Portilla E et al. (2016) Int J Cardiol 212, 174-183. 